\title{
A Music Therapy System for Patients with Dementia who Repeat Stereotypical Utterances
}

\author{
Chika Oshima $^{1,2, a)}$ NaOKI Itou ${ }^{3, b)}$ Kazushi Nishimoto $^{4, c)}$ \\ Kiyoshi Yasuda ${ }^{5,6, d)}$ NaOHIto Hosoi $^{7}$ Hiromi Yamashita ${ }^{8, \dagger 1}$ \\ Koichi NaKaYama $^{9, \text { e) }}$ Etsuo Horikawa ${ }^{2}$ \\ Received: June 27, 2012, Accepted: January 11, 2013
}

\begin{abstract}
Some patients with dementia repeat stereotypical utterances and/or scream in agitation for several hours. Music therapy is a method known to alleviate the symptoms of dementia. Altshuler explained that a music therapist should first play music that matches the current mood of a patient according to the iso-principle, principle of music therapy. We thought that if certain types of music can calm patients down, a music therapy system that is usable for musical novices could be useful in nursing homes. Therefore, we present a music therapy system, "MusiCuddle," that automatically plays a short musical phrase (tune) in response to a caregiver's simple key entry. This music overlaps with patients' utterances and/or screaming. The first note of the tune is same as the fundamental pitch (F0) of the patient's utterances. We compiled four types of tunes (chords, cadences, Japanese school songs, and phrases created from the patients' utterances) into a database. The cadences were selected from established music scores and began with an unsteady or/and agitated chord in order to resonate with the patient's mental instability. We conducted a case study to investigate how MusiCuddle changes a patient's behaviors. In the case study, the pitches extracted from the patient's utterances were varied and wide-ranging. We thought her level of agitation might be reflected in her pitches. Pitch differences in the first note affect and change the entire mood of the music. Therefore, it may be said that the MusiCuddle can play music to resonate with his/her mood by extracting pitch from her utterance in accordance with the iso-principle. Moreover, we recorded the patient's utterances and compared them with vs. without using MusiCuddle to estimate the influence of MusiCuddle. The results suggested that tunes presented by MusiCuddle may give patients an opportunity to stop repeating stereotypical utterances.
\end{abstract}

Keywords: dementia, BPSD, music therapy, iso-principle, case study

\section{Introduction}

Dementia is the deterioration of cognitive ability and skills due to an organic disorder. The Ministry of Health, Labour and Welfare estimates that the number of patients with dementia in Japan is 3.05 million [1]. There are more than 70 causative diseases of dementia. Typical diseases that cause dementia are Alzheimer's, dementia with Lewy bodies, cerebral vascular de-

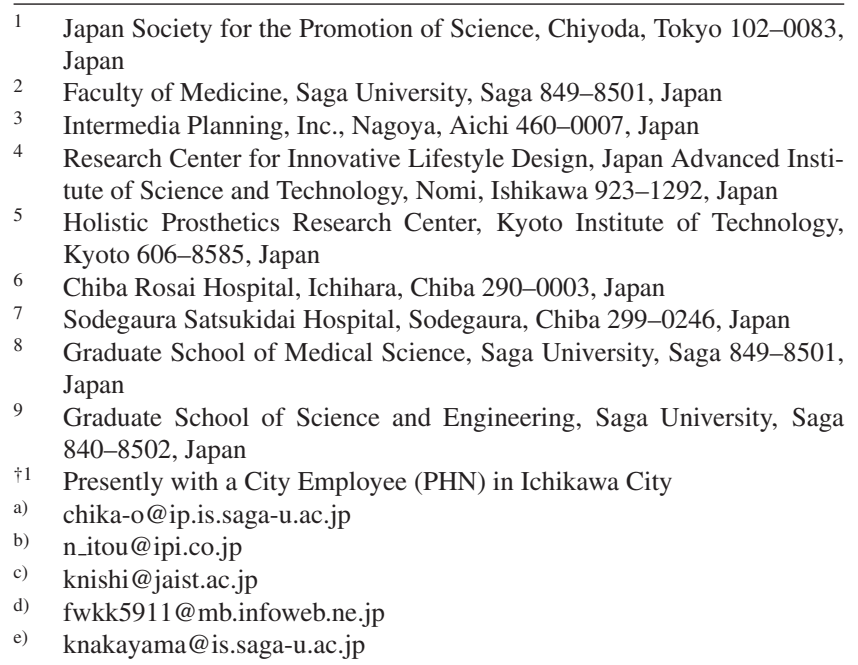

mentia, and frontotemporal dementia (FTD). The symptoms of dementia are divided into core symptoms and behavioral and psychological symptoms of dementia (BPSD). BPSD includes agitation, aggression, wandering behavior, hallucinations, and delusions. However, appropriate care is thought to alleviate and slow the progression of these symptoms.

Music therapy is a method known to alleviate the symptoms of dementia. Park et al. conducted an experiment in which individuals with dementia listened to their preferred music. The results showed that mean agitation levels were significantly lower during than before listening [2]. Another study showed a significant reduction in activity disturbances, aggressiveness, and anxiety in a group assigned to music therapy [3]. The study of Nair et al. found that ambient (soft and slow) Baroque music did not have a calming effect. Therefore, in order to achieve the desired behavioral effect, music may need to be tailored rather than generalized [4].

One symptom of BPSD is repetition of stereotypical utterances and/or screaming for many hours with agitation. Although doctors, nurses, and caregivers can talk to patients with BPSD and have them focus on other types of behaviors, it is not easy to calm them down. Our opinion is that music could calm them. Therefore, a music therapy system that can be presented by a musical novice could be useful in nursing homes. 
The iso-principle [5] is well known to music therapists trained in the United States [6]. "Iso" simply means "equal," that is, the mood or the tempo of the music must initially have an "iso" relationship with the mood or tempo of the patients. The principle extends to volume and rhythm [5]. The actual use of music in a particular therapeutic situation depends upon the particular needs of the individual, such as the need for an immediate change in patient' behavior to relieve troubling conditions [7]. For example, if the patient is distressed or agitated, then the quality of the music should initially match his or her mood and energy. When the iso-principle is adopted, the first choice of music may be familiar and energetic [8]. The therapist's music can be matched with the patient's music: "match" means that the therapist's music is not identical to the patient's music, but it is the same in style and quality [9]. Likewise, in "validation," methods used for communication between patients and therapists, a therapist observes the mood of a patient and emits the same tone with his/her voice to indicate that he/she sympathizes with the patient [10]. This type of therapy may be also effective for BPSD, especially repetitive utterances and/or screaming.

However, it would be difficult to assign a music therapist to every nursing home. Our opinion is that if even caregivers who are musical novices, could address these symptoms of dementia with music, their patients would become calmer and happier.

In this paper, we present a music therapy system, "MusiCuddle," that semi-automatically plays a short musical phrase (tune) that overlaps with patient's utterances and/or screaming. This system continuously extracts fundamental pitches (F0) from the patient's utterances. In response to an operator's (e.g., a caregiver's) simple key entry, the system determines a pitch at a predetermined interval. The system selects a tune from the database on the basis of that extracted pitch. The first note of the tune is the same as the F0 of the patient's utterance. We expect that this function may resonate with the patient's emotions in accordance with the iso-principle.

We compile four types of tunes in MusiCuddle. One type is comprised by "cadences" that begin with dissonant chords and finish with consonance chords. Generally, dissonant chords indicate tension, and consonant chords indicate relaxation. According to the iso-principle, this type of tune begins with a chord that resonates with the patient's agitated mood and finishes with a chord that calms his/her mood. Moreover, the 96 included cadences are of two types: the first type of cadence begins with an extremely agitated chord, and the other type begins with a milder (but still unsteady) chord. The operator (caregiver) can easily choose from these types depending on the patient's condition.

We conduct a case study to investigate how MusiCuddle changes a patient's behaviors. We think that music presented through MusiCuddle disrupts the participant's problematic utterances. Therefore, we compare the participant's utterances while listening to the music with those without music. Especially, we focus on the fact that the participant stuttered: if the music diverted the participant's attention from her repetitive stereotypical utterances, she would be expected to stutter.

In the next section on the related works, we introduce previous music therapy systems. Section 3 illustrates examples of patients who repeat stereotypical behavior and utterances. Section 4 describes the processing employed by MusiCuddle, and Section 5 describes the database of MusiCuddle in detail. Section 6 illustrates experiments undertaken by the author, who presented tunes to a patient with dementia using MusiCuddle. In Section 7, we discuss MusiCuddle's contribution to the reduction of the patient's symptoms. Section 8 concludes this paper and outlines future works.

\section{Related works}

Music therapy systems for patients with dementia have been investigated. "Media Memory Lane [11]" and "Picture Gramophone [12]" are systems that present old popular songs and display old video pictures. Kuwahara, et al. presented an authoring tool to assist in the production of a reminiscent video [13]. Music is also presented with reminiscent video. "CIRCA [14]" is structured to promote patients' speech by reminiscence. When a patient with dementia touches a photograph on the screen of CIRCA, music suited to the photograph is presented. "Express Play [15]" is a support system that patients with dementia compose music.

These systems are said to be useful in reducing the aggression of patients. However, these systems are only useful for early to non severe stage of dementia. Users of these systems can watch photographs, television, and sit in front of a screen, which they can touch at will. On the other hand, MusiCuddle is developed as a support system for a caregiver. Even if the caregiver is a musical novice, he/she can present music to patients with early to severe dementia. Moreover, we consider MusiCuddle reflects an expression (utterance) of each patient in music.

\section{Patients Who Repeat Stereotypical Behav- iors and Utterances}

In this section, we show some examples of patients who repeat stereotypical behavior and utterances. We recorded their utterances with mental instability for one month. Table 1 shows their symptomatic state, the cause of their dementia, their daily attitude, and the situation at the time of recording. The term "HDS-R (Revised Hasegawa's dementia scale) [16]" in the table refers to an intelligence test consisting of 9 simple questions, with a maximum score of 30 . A score over 21 score identifies a person who is not a patient.

The scores in the table show that most of these patients had severe dementia. Even patients who had mild dementia (i.e., Patients C and I) showed mental instability and repeated stereotypical utterances. The examples show that the patients uttered a strange noise with emotion, calling for someone who was not there or for a caregiver. Even after the caregiver responded to their requests, several of them repeated the same requests, as seen with Patient A. Patient I locked herself in a restroom when she was agitated. She repeated stereotypical utterances in a loud voice all day. However, when a caregiver says something to her, she can greet clearly.

In most cases, we could not understand what the patients were saying. Although doctors, nurses, and caregivers talked to the patients and led them to another action, it was not easy to calm 
Table 1 Data of stereotypical utterances with mental instability.

\begin{tabular}{|c|c|c|c|c|c|}
\hline \multirow[t]{2}{*}{ Patient } & Cause of dementia & Sex & Age & HDS-R & Daily attitude \\
\hline & \multicolumn{5}{|c|}{ Situation at the time of recording } \\
\hline \multirow[t]{2}{*}{ A } & Alzheimer & female & 89 & 7 & She cries "I want to go to the restroom!" \\
\hline & \multicolumn{5}{|c|}{ She repeated "Neeee" in a falsetto. } \\
\hline \multirow[t]{2}{*}{ B } & Cerebrovascular & male & 58 & 3 & Irritable. Act menacingly. Behave rudely. \\
\hline & \multicolumn{5}{|c|}{ He repeated "Try not to bother someone," "Please" in tears. } \\
\hline \multirow[t]{2}{*}{$\mathrm{C}$} & Cerebrovascular & female & 77 & 20 & Little memory problem, but repeats same words in loud voice. \\
\hline & \multicolumn{5}{|c|}{ She repeated "ttaka?(Did you do it?)" in a falsetto. } \\
\hline \multirow[t]{2}{*}{ D } & Alzheimer & female & 69 & Inoperativeness & She utters meaningless sounds. \\
\hline & \multicolumn{5}{|c|}{ She cried "Aaaaa" in a loud whisper. } \\
\hline \multirow[t]{2}{*}{$\mathrm{E}$} & Alzheimer & female & 78 & Inoperativeness & Severe symptoms. \\
\hline & \multicolumn{5}{|c|}{ She repeated "Tasukete(Help me!)" in a loud voice. } \\
\hline \multirow[t]{2}{*}{ F } & Alzheimer & female & 90 & Inoperativeness & She wanders around in crying in a loud voice. \\
\hline & \multicolumn{5}{|c|}{ She repeated meaningless sounds thickly. } \\
\hline \multirow[t]{2}{*}{ G } & Alzheimer & male & 78 & Inoperativeness & Irritable. Cries in a loud voice. \\
\hline & \multicolumn{5}{|c|}{ He uttered meaningless sounds. } \\
\hline \multirow[t]{2}{*}{$\mathrm{H}$} & Alzheimer & female & 82 & Inoperativeness & She always utters in a loud voice. Poor communication. \\
\hline & \multicolumn{5}{|c|}{ She talked in anger and in tears. We cannot understand what she said. } \\
\hline \multirow[t]{2}{*}{ I } & Frontotemporal & female & 70 & 17 & Good communication. \\
\hline & \multicolumn{5}{|c|}{ She locked herself in a bathroom, and repeated "Imasen desuyo (I am not here)" in a loud voice. } \\
\hline \multirow[t]{2}{*}{$\mathrm{J}$} & Lewy bodies & female & 83 & 13 & Irritable. \\
\hline & \multicolumn{5}{|c|}{ She repeated "Bakayaro (Stupid)" with a furious voice. } \\
\hline
\end{tabular}

them down. Therefore, music that could resonate with the patients' emotions and calm their symptoms would be expected to help the caregivers in caring for these patients.

\section{System Set-up}

Figure 1 shows a user interface of MusiCuddle for a caregiver. MusiCuddle is a music therapy system that presents music when an operator (e.g., a caregiver) pushes any of the keys of the electronic keyboard or a button on the interface of the system. Previously, we have to select a folder of tunes and move it into the same folder as MusiCuddle. We can select from some types of folders: cadences, chords, songs (see Section 5). Once the play button is pushed, the system continuously extracts pitches (F0) from sounds (the patient's utterances). When the operator pushed the trigger's button again, the system determines a pitch at a predetermined interval. Then, the system selects a tune file in the database on the basis of that extracted pitch. The first note of the tune is the same as the pitch extracted from the patient's utterances.

We show two examples. A user has selected the folder of "Cadence." The system extracts a pitch from patient's utterances, $\mathrm{C} 4$. Then, the system selects one of cadence, of which first note is $\mathrm{C} 4$. In other example, a user has selected the folder of a song, "Yuki" phrase. The system selects Yuki phrase in F major that the first note is $\mathrm{C} 4$.

We employ a pitch extractor to extract pitches (i.e., C, D, E) from the patient's utterances. This is based on the technique for extraction of sounds that have unstable pitches and unclear periods, such as natural ambient sounds and the human voice, into musical notes [17].

In the original system shown by [17], if the operator gave a start trigger, the system would initiate the processing to obtain the F0 (fundamental frequency) time series from the acoustical signals (i.e., a singing voice), which were being recorded via the microphone. The short-term F0 estimation by Fast Fourier Transform (FFT) and Inverse Fast Fourier Transform (IFFT) for the power

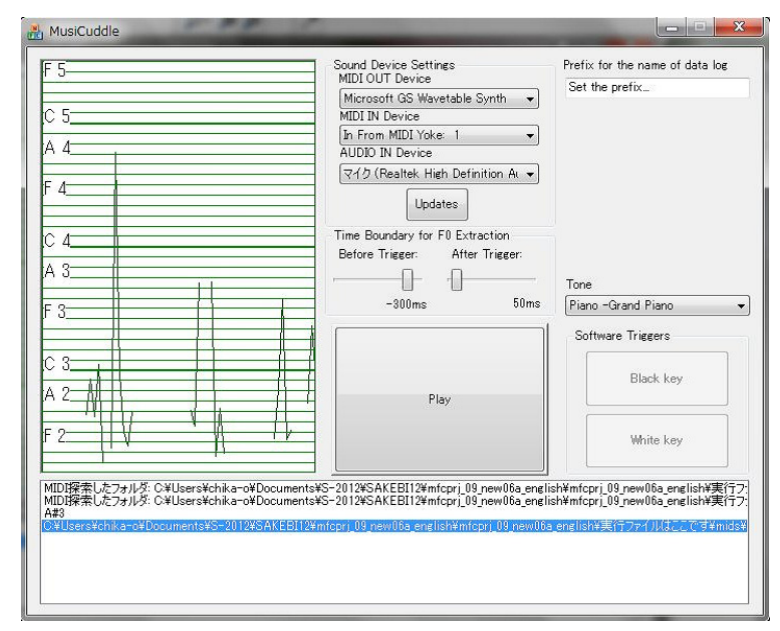

Fig. 1 User interface of MusiCuddle for a caregiver.

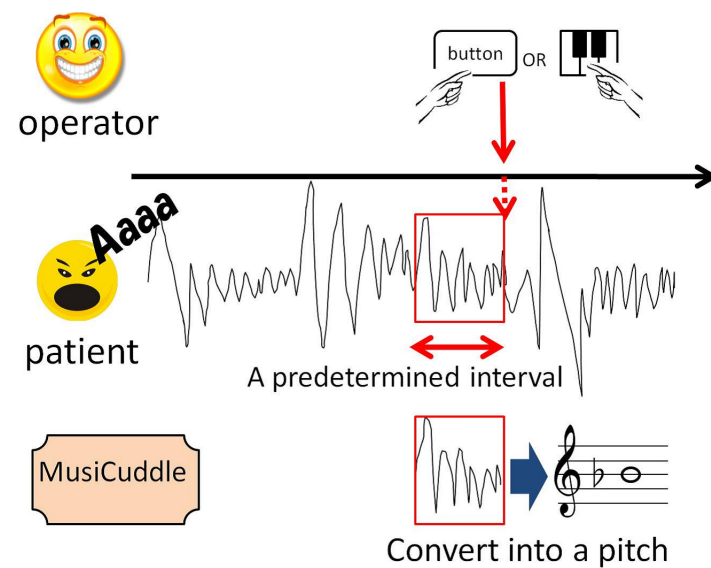

Fig. 2 How to convert an utterance into a pitch.

spectral is repeated until the system catches an end trigger from the operator (i.e., the caregiver). The system then calculates a histogram of pitches with the F0 time series between the start and end triggers. Finally, only the most frequent pitch is selected and is output as the pitch of the period.

For our research, some processing designs were modified. Fig- 
Table 2 Tunes.

\begin{tabular}{c|l|l}
\hline \hline Types & \multicolumn{2}{|l}{ Details } \\
\hline \multirow{2}{*}{ Four chords } & Interval & Perfect prime, Perfect fifth (consonant interval), Major seventh (dissonant interval) \\
\cline { 2 - 3 } & Rhythm & Quarter note, Eighth note, Sixteenth note, Random \\
\cline { 2 - 3 } & Change of volume & No change, Crescendo, Decrescendo \\
\hline \multirow{2}{*}{ Cadence } & Bach & Das Wohltemperierte Klavier Band 1, BWV846-869 \\
\cline { 2 - 3 } & Shostakovich & 24 Preludes and Fugues, Op.87 \\
\hline Japanese school song & "Yuki," "Akaikutsu," "Hana," "Tsukinosabaku," etc \\
\hline Stereotypical utterance & Ex. Utterance, "i-ma-se-n-de-su-yo" is converted into a phrase "re-re-re-si-la-la-si." \\
\hline
\end{tabular}

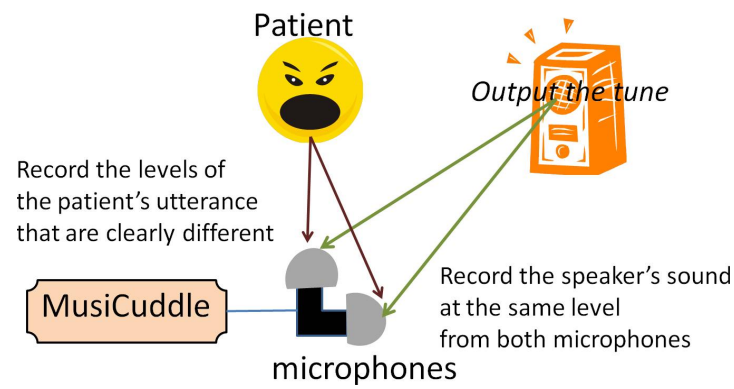

Fig. 3 Calculates the differential signals from both microphones to cancel the sounds of cadence.

ure 2 shows the processing of the system. Considering the attitude of the operator, we would assume that the triggers would be input after the operator catches the utterance of the patient. Therefore, we omitted the start trigger. The system starts a shortterm F0 estimation just after invocation of the system and continues it thereafter. When the operator inputs a trigger that is regarded as an end trigger, the system calculates a representative pitch for a predetermined period just before the trigger based on the above-mentioned method. Then the system plays a prepared MIDI (Musical Instrument Digital Interface) sequence that corresponds to the representative pitch. These modifications of our system improve usability by reducing the time lag between the input of the trigger and the output of phrase.

To extract the F0 against the mixed acoustical signal of the patient's utterance and the tune output from the speaker, our system needs two of the same microphone (ideally one stereo microphone) and one speaker. Figure 3 shows the setting of the microphones. The microphones are set in front of the speaker to record the speaker's sound at the same level from both microphones. On the other hand, both microphones are displaced against the patient to record the levels of the patient's utterance that are clearly different. The system calculates the differential signals from the signals of both microphones to cancel the sounds of the MIDI sequence where they are localized in the center position. The F0 estimation is then determined with these differential signals.

\section{Database of tunes}

We tentatively compiled the four types of phrases in the database. Table 2 shows some details of the phrases. Patients who repeat stereotypical utterances often experience agitated moods and high anxiety. Generally, dissonant chords indicate tension, and consonant chords indicate relaxation. Clinically, a combination of these chords can elicit tension and relaxation within the human body: therefore, we prepared both dissonant

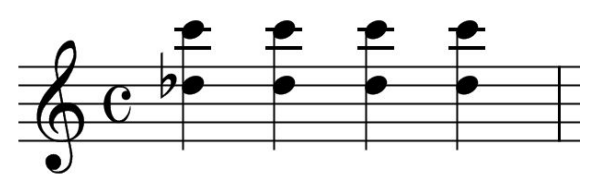

Fig. 4 Four repetitions of the same chord are presented in one phrase.

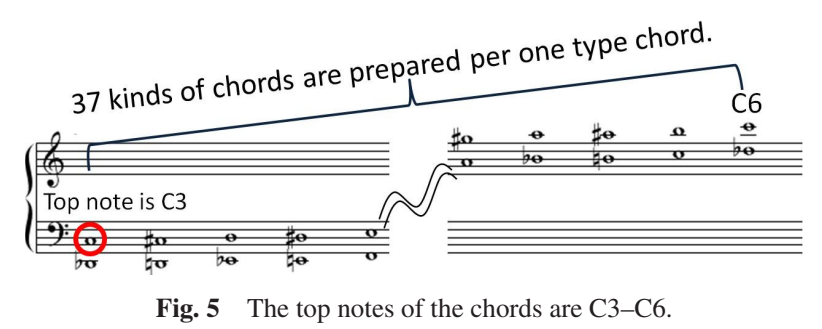

and consonant chords. Moreover, we prepared cadences that begin with dissonant chords and finish with a consonant chord.

\subsection{Four Chords}

"Four chords" means that four repetitions of the same chord are presented in one phrase (see Fig. 4). The chord is composed of two notes. Thirty-seven MIDI files, in which the top notes of the chords are C3 C6, were prepared per type of chord (see Fig. 5). Accordingly, the system can select a MIDI file of which the top note of the chord is similar to the patient's F0. There are 1,332 MIDI files (phrase files) for the four-chords type of phrase. The types of chords consist of 3 different intervals for each pitch, 4 different rhythms, 3 types of dynamic changes, and 37 different pitches for each chord.

The "Interval" column shows the intervals between the two notes comprising each chord. In general, a chord consisting of consonant interval is a consonant chord, and a chord that includes dissonant interval is a dissonant chord. A perfect prime and a perfect fifth are consonant intervals, and a major seventh is a dissonant interval. In musicology, the larger the ratio between two frequencies, the more dissonant the chord consisting of those two pitches becomes. The frequency ratio of a minor seventh interval $(9: 16)$ is larger than that of a major seventh interval (8:15) in equal temperament. However, the error with which people evaluate the dissonance of minor sevenths is larger than that for major sevenths [22]. Previous research in which children drew pictures in response to four musical intervals showed that the major seventh was associated with pictures involving aggressiveness and danger [23]. Therefore, we employ the major seventh as a dissonant chord.

In the "Rhythm" column of Table 2, "Quarter note" means that 
each of the four chords is presented in rhythm at 60 beats per minute. "Random" is unregulated rhythm [18]. In the "Change of volume" column, "No change" means that the four chords are presented at the same volume. "Crescendo" means that the volume of notes increases throughout the presentations of the chord, while "Decrescendo" means that the volume of notes decreases.

\subsection{Cadence}

For the "Cadence" type, we prepared 96 kinds of cadences that begin with dissonant chords and finish with a consonant chord. These cadences were excerpted from two piano suites, "Das Wohltemperierte Klavier Band 1," BWV846-869, composed by Bach, J.S. in 1722, and "24 Preludes and Fugues," op.87, composed by Shostakovich, D.D. in 1952. Both suites consist of 24 pieces in 24 different tonalities (the 12 major and 12 minor keys), and each piece consists of both a prelude and a fugue. Therefore, we can prepare at least 96 cadences if two cadences are chosen from each piece (one from the prelude, and one from the fugue).

Although the study of Nair et al. found that ambient Baroque music did not have any calming effect [4]. They used ambient Baroque music (which is stable, gentle, slow, and soft). The kind of music may not be appropriate for the participating patients according to iso-principle. However, cadence that begin with dissonant chords are not gentle but agitated. Moreover, we needed 12 types of cadences whose top notes of the initial chords are different tones, i.e. C, C\# (Db), D, D\# (Eb), E, F, F\# (Gb), G, G\# $(\mathrm{A} b), \mathrm{A}, \mathrm{A \#}(\mathrm{B} b), \mathrm{B}$. Since Bach's suite "Das Wohltemperierte Klavier" consists of pieces with 12 different keys, it is convenient to use this work for our purpose.

These MIDI files of cadences were created by one of the authors playing a digital piano (real-time recording method). Then, each MIDI file is translated upward/downward by one, two or three octaves to cover all possible F0 values (C3 C6) derived from the patient's utterance. However, these cadences are not transposed into another key. In general, when composers com- pose music, they select tonality by musical expressive intention. Therefore, we use the cadences in original tonality.

If the original MIDI data begin with a note within $\mathrm{C} 3 \sim \mathrm{C} 6$, it is translated into two or three kinds of MIDI files. If the original MIDI data begins with a note outside of the range of C3 C6, four or five kinds of translated MIDI file were created. As a result, there are 312 total MIDI files for the cadences.

Figure 6 shows the database of the cadences and the process of the system after a key entry by the operator. The 96 kinds of cadences are considered to consist of two types. Shostakovich's tunes conclude with more agitated tones, and more emotional and temperamental cadences, achieved through chromatic inflection, compared with those composed by Bach. Therefore, the cadences selected from the pieces composed by Shostakovich were tentatively designated "Black-type cadences." The cadences composed by Bach begin with milder (but unsteady) chords; we call them "White-type cadences" in this paper. The operator can easily choose from these types depending on the patient's condition. When the operator touches a button for the Black-type (or the White-type) cadences or any black key (or white key) on the keyboard, the Black-type (or the White-type) cadence is played through a speaker.

\subsection{Japanese School Song}

We also prepared phrases from Japanese school songs. Ashida reported that reminiscence-focused music therapy might reduce depressive symptoms in elderly people with dementia [19]. Therefore, we selected short phrases from some songs that would be familiar for elderly people; we also created a simple accompaniment for each phrase. Each phrase is arranged into 37 MIDI files, the first note of which are $\mathrm{C} 3 \sim \mathrm{C} 6$.

Figure 7 shows two kinds of phrase consisting of the same tune, "Akaikutsu." The top line in each begins with $\mathrm{C} 4$, and the bottom line begins with G\#4. The difference in pitch of the first notes affects and changes the entire mood of the music.

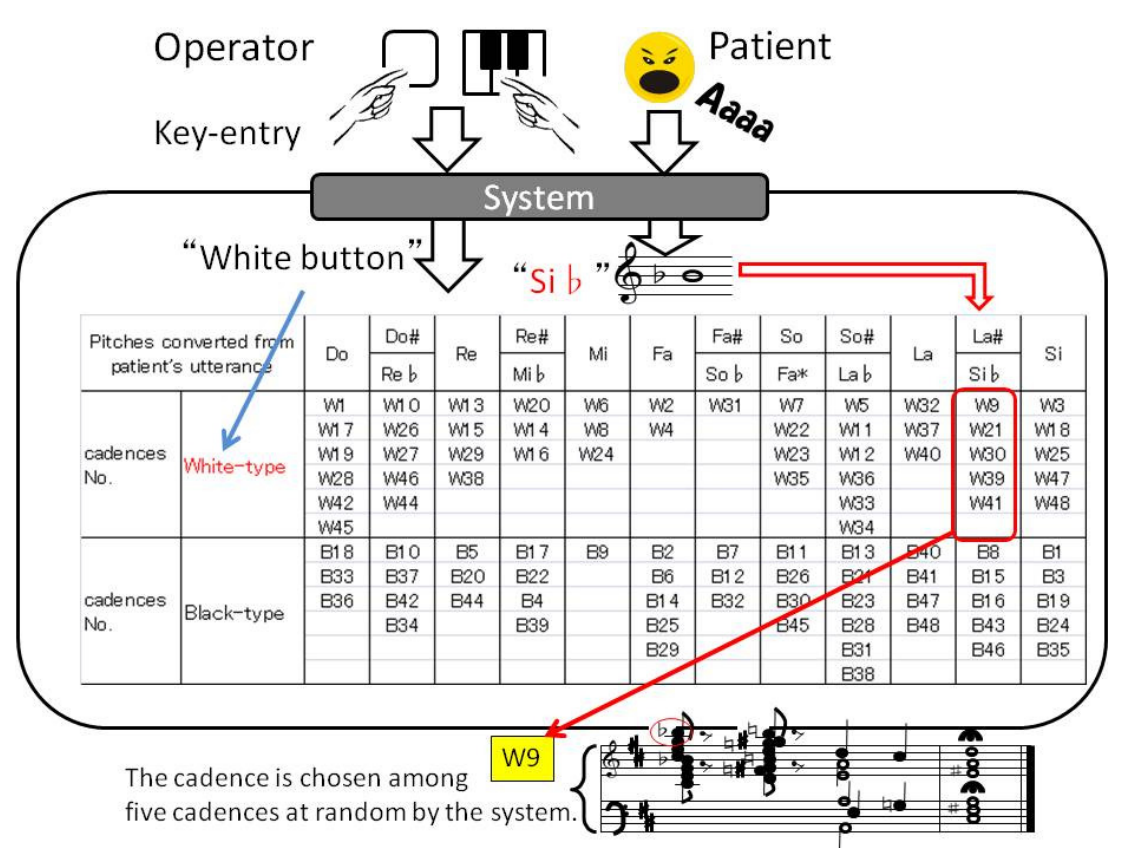

Fig. 6 Selection of a cadence. 
The first note is $\mathrm{C} 4$.

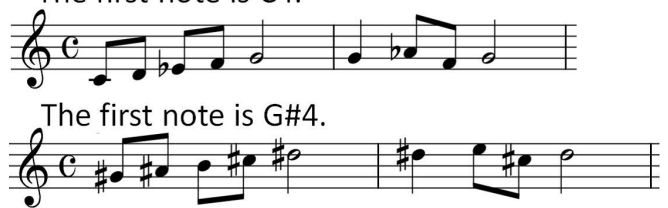

Fig. 7 The difference in pitch of the first note changes the entire mood of the music.

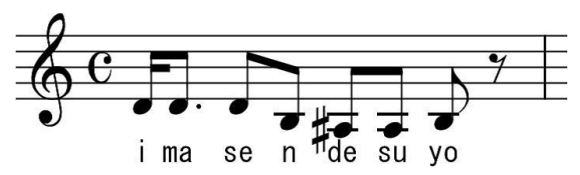

Fig. 8 A segment of the patient's utterance converted into musical notes.

\subsection{Phrase Created from the Patient's Utterances}

The last type of phrases is created from the patient's utterances. All segments of her utterances can be converted into musical notes. Figure 8 shows an example. Previously, her utterance, "i-ma-se-n-de-su-yo [I am not here]," was converted into a phrase "re-re-re-si-la-la-si." This phrase was also arranged into 37 MIDI files, the first notes of which were $\mathrm{C} 3 \sim \mathrm{C} 6$.

\section{Case Study}

We conducted a case study to investigate how a patient's behaviors changed with the application of MusiCuddle. The symptoms of the patient targeted for this study was severe. This case study was conducted for a very short period, and we could enroll only one patient. Therefore, it is not appropriate to make any cognitive assessment [20] or to examine the patient's abilities with respect to activities of daily living [21]. Instead, we record the patient's utterances under conditions with and without the use of MusiCuddle and compare them in order to estimate the influence of MusiCuddle.

\subsection{Ethical Considerations}

This case study was approved by the Research Ethics Board of Saga University. The participant in the case study, who is a patient with dementia; her husband; and the hospital director were informed about the intentions of the case study and the treatment of personal information. Moreover, they were informed that they could withdraw from the case study at any time. Then, we obtained written consent from them.

When we conducted this case study, the hospital director and nurses worked on the same floor and could check on the condition of the participant. If the presentation of sounds from MusiCuddle had not been appropriate and the participant became more agitated, we would have had to abandon the case study immediately.

\subsection{Participant}

The participant was a 72-years-old, hospitalized patient with severe FTD; she is Patient-I in Table 1. She repeats stereotypical utterances for many hours each day. Moreover, when she is agitated, she locks herself in a restroom for a long time while repeating stereotypical utterances. However, she is lucid enough to remember some nurses' names and greet them clearly. She can answer the date and the exact time. Her score on the HDS-R was

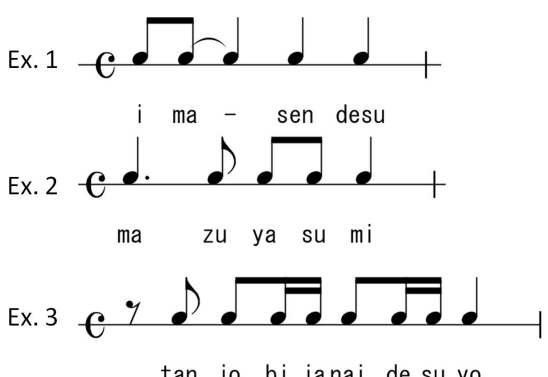

$\tan$ jo bi janai de su yo

Fig. 9 The participant's sentences fit into four-four time.

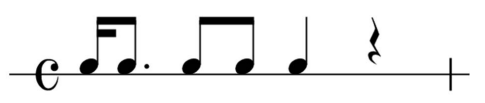

Sentence A: i ma se $\mathrm{n}$ yo

Sentence B: gohan de su yo

Fig. 10 The author repeated sentences in accordance with the participant's repetition.

\section{7 two years ago.}

The following is an example of her usual utterances. This example was uttered in about thirty seconds. "P" means "Participant."

P: haittayo (repeated eight times) mashitayo imasen masen imasendesu masen (repeated three times)

Although she utters many kinds of sentences, most of them are rhythmical and fit into the same meter. Figure 9 describes some examples of her sentences. One of the authors dictated the rhythms of these sentences. These examples show that although the sentences are different, they fit into four-four time.

She repeats stereotypical utterances especially when hungry. She often locks herself in a restroom from around eleven o'clock a.m. until lunchtime, and from around one o'clock p.m. until snack time while repeating stereotypical utterances nearly incessantly. However, she sometimes responds to nurses when they talk to her.

\subsection{Preliminary Experiment}

Adopting the iso-principle, one of the authors attempted to utter according to the participant's utterances. We think that the author's utterances had the same tempo, rhythm, and pitch as those of the participant.

\subsubsection{Method}

When the participant was agitated and repeated the same sentences, one of the authors repeated sentences in the same melody corresponding with the participant's repetition (the same tempo, rhythm, and pitches). Figure 10 shows these sentences in musical notation. Sentence A means the participant's sentence. First, the author tries to repeat the participant's sentences in rhythm. Namely, both of them repeat Sentence A, "i-ma-se-n-yo." Second, the author tries to repeat a different sentence using the same melody as the participant's in rhythm. Namely, the author repeats Sentence B, "go-han-de-su-yo," although the participant is repeating Sentence A.

\subsubsection{Result}

In the first trial, the participant turned around to pay attention to 
the author. However, she kept repeating the same sentence in harmony with the author's repetition. Her utterances became louder. In the second trial, the participant changed from the sentence A to the author's sentence, "go-han-de-su-yo" in the same melody. Then, the participant left the restroom and went toward a table for lunch. In a moment, however, she returned to the restroom and repeated the same sentences.

\subsubsection{Discussion}

When the author repeated sentences in accordance with the participant's repetition and used the same melody, the participant kept repeating the same sentence in a loud voice. The author's utterances could have caused increased symptoms of agitation in the first trial.

\subsection{Method}

\subsubsection{Recording}

We stood by from ten o'clock a.m. to noon and from one o'clock p.m. to half-past two p.m. for 2 days. After this case study, we compared the participant's utterances with music with those without music (see Section 6.5) to estimate the influences of MusiCuddle. Therefore, we set two time periods, one with the use of MusiCuddle and one without MusiCuddle.

During the time with the use of MusiCuddle, we started MusiCuddle and selected a tune. When the participant began to repeat stereotypical utterances, we presented the tunes arbitrarily by giving triggers to MusiCuddle.

\subsubsection{Equipments}

The experiment was conducted in a hospital where the participant was hospitalized. Figure $\mathbf{1 1}$ shows the setting of the case study. The music was presented through a wireless cuboidal speaker with Bluetooth, which measured, $123 \times 36 \times 35 \mathrm{~mm}$, and the patient's utterances were recorded through a wireless, columnar microphone with Bluetooth measuring about $75 \mathrm{~mm}$ in height and $24 \mathrm{~mm}$ in diameter. These devices were set on the door of the restroom.

Our system requires two of the same microphone (one stereo microphone). When the operator inputs the trigger, even when the previous tune is being presented, the system extracts F0 against the mixed acoustical signal of the patient's utterance and the tune being presented from the speaker. However, it is not so safe to use the stereo microphone in the hospital, because it is large in size and wired to its receiver. Thus, we did not use the stereo microphone in this experiment, and the operator did not input triggers when the previous tune was being presented.

\subsubsection{How to use MusiCuddle}

When the participant is agitated and repeats stereotypical utterances in a restroom, an operator (one of the authors) presents music by using the MusiCuddle system in front of the restroom. The operator listens to the patient's utterances outside of the restroom. When the operator finds a period during which the patient utters an almost stable pitch, she clicks the trigger button. Once the trigger button has been clicked, a tune is retrieved from the database on the basis of the detected pitch, and it is automatically performed to overlap with the participant's utterances. In this case study, we prepared seven type of tunes: Four chords (Major seventh, Quarter note, and No volume change), Cadence, "Yuki," "Akaikutsu," "Hana," "Tsukinosabaku," and Stereotypical utterance (i-ma-se-n-yo). All of the tunes consist of very short phrases lasting 3 30 seconds. The operator selected tunes considering the participant's reactions and condition. The operator clicked the trigger button again to perform the next tune when the performance of the current tune ended.

\subsection{Analysis Method}

In this case study, we investigate how MusiCuddle influences the patient's stereotypical utterances. If the music presented from MusiCuddle distracted the patient's attention from her stereotypical utterances, her utterances would be disrupted and she would stutter. Therefore, we compare the participant's utterances while listening to music with those without music. Especially, we focus on the patient's stuttering to detect distraction of the patient's attention.

The participant's utterances are segmented into small sentences according to the method of repetition. One of the authors decided segmentation points according to the meanings of utterances by reference to the participant's breathing. For example, the following utterances (P1) are segmented like the next line (P2).

P1:imasendesuimasendesuhitoyasumimazuyasumiimasendesuyo P2:imasendesu (not being here) / imasendesu (not being here)

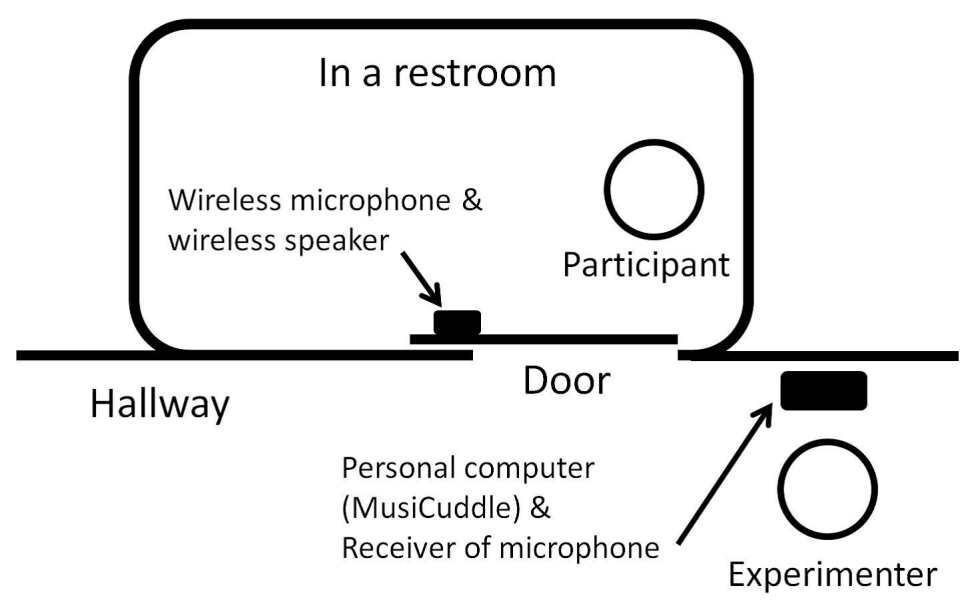

Fig. 11 A small wireless speaker and microphone are set on the door of the restroom. 


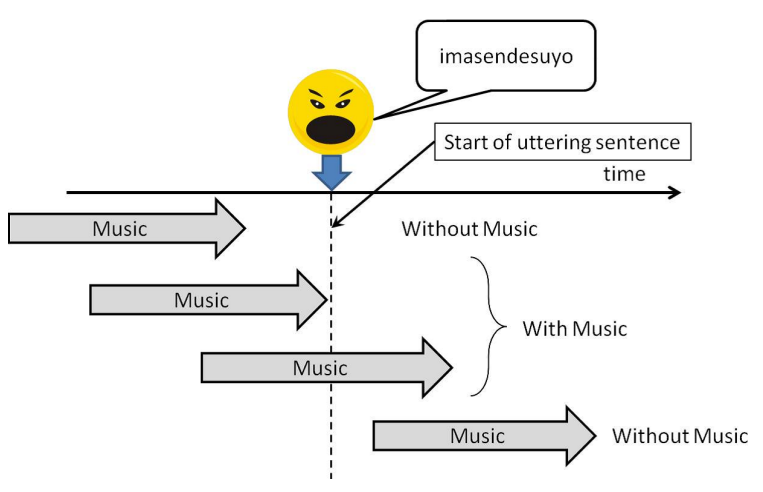

Fig. 12 Determination of whether each sentence was uttered "with or without music."

/ hitoyasumi (taking a rest) / mazuyasumi (taking a rest) / imasendesuyo (not being here)

Then, we analyzed the influences of the music on the patient's utterances. First, we determined whether each sentence was uttered "with music" or "without music" on the basis of the following conditions (see Fig. 12):

(1) If the patient uttered a sentence while a tune was being performed, the sentence was considered to be uttered with music.

(2) If the patient began to utter a sentence just after a tune had finished, the sentence was considered as being uttered with music.

(3) If a tune started after the patient had started uttering a sentence, the sentence was considered to be uttered without music.

(4) Otherwise, the sentence was considered as being uttered without music.

In the following example, the tune was presented in the middle of "hirugohandeha (fragment of "It is not lunch time")." Therefore, "masende (fragment of "not being here")" and "hirugohandeha" were considered as being uttered without music, while "imasendesu (not being here)," "ima (fragment of "not being here")," and "gohanden (fragment of "It is not lunch time")" were considered as being uttered with music.

P: masende hirugohandeha imasendesu ima gohanden

$$
\text { [ }
$$

In this case, we consider that the sentence "hirugohandeha" was unaffected by music. Moreover, we consider that "gohanden" was affected by the music, because this sentence started to be uttered immediately after the presentation of the music.

Next, we find sentences on which the participant stuttered. She often repeats several stereotypical sentences without any slight changes many times (see Section 6.2). However, if the music distracted her attention from her repetition of stereotypical utterances, she stuttered, uttering only a part of stereotypical sentence or a sentence different from a stereotypical sentence. Therefore, if we find such a sentence including words that were part of an immediately preceding sentence (but not exactly the same as the immediately preceding sentence), she was considered to have stut- tered.

We determined whether each sentence included words that were part of the immediately preceding sentence. In the following example, we consider that she stuttered, as "ima" is included in the immediately previous sentence "imasendesu:"

P: imasendesu ima

\subsection{Results}

\subsubsection{Intended Records}

The intended records documenting our analysis of the participant's utterances constitute only three parts of the entire recorded dataset; the lengths of the three parts are 16, 8, and 3 minutes. Although we recorded for a much longer time period, we could not use the other parts because of extraneous noises that masked the patient's utterances. It seems that the restroom's iron door blocked communication between the wireless microphone and the personal computer. Moreover, we could not record when the participant moved to some unexpected rooms. Therefore, there is a large gap between the time of using MusiCuddle and the total recording time.

Of the 27 minutes of intended records, the total time of tune presentation was 6 minutes and 54 seconds, approximately onefourth of the total recording time. For the first and second recording sessions, we presented the tunes using MusiCuddle. In contrast, we did not present tunes at all during the third recording session.

\subsubsection{Presented Tunes}

Table 3 shows the kinds of tunes, times of the tunes, and total time of tune presentation during the first and second recording sessions. In the first recording session, we first presented six four chords. At this time, we used one type of four chords: Major seventh, Quarter note, and No volume change. This chord can convey an unsteady image to the participant. Next, we presented a Japanese school song, "Yuki," four times. The patient sang only the last phrase, "zun zun tsumoru [accumulate rapidly]" along with the melody the first time. Continuously, "Akaikutsu" was presented five times. Next, five cadences were presented. The kinds of cadences were determined by the F0 values derived from her utterances (see Fig. 6). After that, we again presented "Yuki," during which she sang again. The last tune of the first recording session was a Japanese school song, "Hana."

In the second recording session, first, we presented a Japanese school song, "Tsukinosabaku," four times. Then, "Yuki" was presented nine times continuously; however, she did not sing along with those presentations. Next, we presented the phrase created from her utterance "i-ma-sen-de-su-yo (see Fig. 8)" five times. At the end of the recording, we presented "Hana" two times.

The "Beginning note" column shows the first note of each tune as well as the F0 values derived from the participant's utterances. The first notes of 49 tunes are contained within D3 D4 (one octave). Only one tune began with G\#4 (the tune was "Hana."). This pitch, G\#4, is much higher than D4; at the time, when this pitch was derived from the participant's utterance, she was very agitated because she was hungry. 
Table 3 Presented tunes in the experiment.

\begin{tabular}{c|c|c|c|c|c}
\hline \hline Types & Tunes & Time(sec.) & Number of times & Beginning note & Total time(sec.) \\
\hline \multirow{4}{*}{ Four chords } & $\begin{array}{c}\text { Major seventh \& } \\
\text { Quarter note \& } \\
\text { No volume change }\end{array}$ & 3 & 6 & D3(2),D\#3,F3,A3,C4 & 18 \\
\hline \multirow{2}{*}{ Cadence } & No.22 Fugue (B) & 8 & 1 & C4 & 8 \\
& No.1 Fugue (B) & 8 & 1 & F3 & 8 \\
& No.15 Fugue (B) & 11 & 1 & F\#3 & 11 \\
& No.22 Prelude (S) & 15 & 1 & D4 & 15 \\
& No.3 Fugue (S) & 25 & 1 & F\#3 & 189 \\
\hline Japanese school song & "Yuki" & 9 & 21 & D\#3, E3(3),F3(7),G3(2),G\#3,A3(3),A\#3,B3,C4,D4 & 50 \\
& "Akaikutsu" & 10 & 5 & D3,F3,F\#3,G\#3,A\#3 & 32 \\
& "Hana" & 8 & 4 & D3,G\#3,A\#3,G\#4 & 44 \\
\hline Stereotypical utterance & i-ma-se-n-de-su-yo & 3 & 5 & F\#3,F3, C4,C3 & 15 \\
\hline Sum & - & - & 50 & G3,G\#3,A3(2),A\#3 & - \\
\hline
\end{tabular}

In the "Tunes" column, "(B)" means that the tune is part of a piece composed by Bach, and "(S)" means that the tune is part of a piece composed by Shostakovich. In the "Beginning note" column, C, D, E A, and B represent Do, Re, Mi La, and Si. A4 is $440 \mathrm{~Hz}$. The values in parentheses show the number of occurrences of each beginning note.

Table 4 Sentences segmented from the participant's utterances in the case study.

\begin{tabular}{|c|c|}
\hline Estimated meaning & Sentences \\
\hline I am (not) here. & $\begin{array}{l}\text { imasu (1), imasendesu (201), imasen (48), imasende (43), ima (5), deimasendesu (1), sokoniimasen (1), imasenyo (1), } \\
\text { uruchiimasendesu (1), ryugaimasende(1) }\end{array}$ \\
\hline It is (not) lunch time. & $\begin{array}{l}\text { mazugohandesu (78), mazugohan (14), mazugohande (6), hirugohannarimasendesu (5) } \\
\text { gohandesu (4), hirugohandashimasendesu (2), hirugohannarimasende (2), gohandashimasendesu (2) } \\
\text { haisugugohandashimasendesu (2), mazugohandesuyo (2), gohan (2), gohanden (1), gohannarimasendesu (1) } \\
\text { gohannaidesuyo (1), hirugohande (1), gohandashimasende (1), gohanninarimasu (1) } \\
\text { gokaimenogohandashimasendesu (1), mawarinogohangoyamoyashisendesu (1) }\end{array}$ \\
\hline First, & mazu (34), mazuyasumi (33), mazudesu (32), ma (6), mazuya (3), mazudesu (2), mazude (1), mazugo (1), mazuyasu (1) \\
\hline Not do & masende (10), masendesu (6), masendesuyo (2) \\
\hline Bath time, Break & ofurohaittadesuyo (2), ofuro (1), ofurojaimasende (1), hitoyasumi (1) \\
\hline (Not) Birth day & $\begin{array}{l}\text { tanjobijanaidesu (3), tanjokainaidesuyo (3), tanjobijanaidesu (1), tanjobijaimasendesuyo (1) } \\
\text { tanjobijaarimasendesu (1), tanjobijaarimasendesuyo (1) }\end{array}$ \\
\hline Time & $\begin{array}{l}\text { 1ji40fundesuyo (13), yoruninarimasendesu (9), 1jihandesuyo (8), 3jihanninarimasendesu (8), yoruninarimasendesuyo (5) } \\
\text { handesuyo (4), 3jihandesuyo (4), 1jihande (3), 2jihandesuyo (2), 3jininarimasendesuyo (2), 1jihandesune (1) } \\
\text { 1ji10fundesune (1), 1ji (1), 1jihandesuyo (1), 3jihanni (1), 3jihanninarimasendesuyo (1), mou3jininarimasendesu (1) }\end{array}$ \\
\hline Snack time & oyatudesuyo (1), oyatujaimasendesu (1), keikihanaidesuyo (1), keikihanaidemasendesu (1), keikihanaitodesu (1) \\
\hline Soon & suguha (1), suguhanaidesu (1) \\
\hline "Yuki" & zunzuntumoru (2) \\
\hline Question & imashitaka (1) \\
\hline Greeting & konnichiha (1) \\
\hline Others & dojoninarimasende (1), ugoninarimasende (1), sonouchimasende (1), mashi (1), bokujaarigatoarigato (1), basyohanaidesuyo (1) \\
\hline
\end{tabular}

Values in parentheses show the numbers of times each sentence was uttered.

\subsubsection{Classify Utterances}

The participant emitted utterances at all times during the experiment. Table 4 shows the kinds of sentences. Six hundred eighty sentences were segmented (84 kinds) in 27 minutes. The most uttered sentence was "imasendesu (201 times)."

In many cases, the contents of the sentences were almost the same, even when their constituent words varied slightly. For example, "imasen" and "imasendesu" have the same meaning, "I am not here." Moreover, in certain instances, only parts of sentences were uttered ("ima," "imasende").

She repeated the same words many times, uttered different words in sequence, or uttered slightly different words continuously both in the rhythm of the presented-music and not. In the following example, she repeated the same words many times:
P: mazuyasumi mazuyasumi mazuyasumi mazuyasumi...(total number of repetitions was seven)

In the following example, she uttered different words in sequence:

P: gohandashimasendesu mazu imasendesu oyatuja imasendesu mazuyasumi

In the following example, she uttered slightly different words continuously:

P: imasende imasendesu imasen imasendesu ima imasendesu 
Table 5 The numbers of changes in repeated sentences.

\begin{tabular}{c|c|c}
\hline \hline & with music & without music \\
\hline $\begin{array}{c}\text { changing sentence } \\
\text { (ALL) }\end{array}$ & 114 & 179 \\
\hline $\begin{array}{c}\text { include the words of } \\
\text { the immediately previous sentence. }\end{array}$ & 94 & 74 \\
\hline rate $(\%)$ & 82.5 & 41.3 \\
\hline
\end{tabular}

Most of the sentences were rhythmical and fitted into four-four time (see Fig. 9). However, short sentences such as, "ima" and "mazu" fitted into four-one (irregular) time.

Table 5 shows the comparison between "with music" and "without music." The numbers of different sentences uttered were 114 with music and 179 without music. The total recording time was 27 minutes, and tunes were presented for 6 minutes $54 \mathrm{sec}-$ onds of that time. Changes in the sentences uttered by the participant numbered about 16 per minute with music and 9 per minute without music. Therefore, we can say that the participant changed her utterances more often with than without music.

Next, we determined whether each sentence included words that were part of the immediately preceding sentence in order to determine on which sentences the participant stuttered. The results indicated that with music, 94 out of 114 sentences $(82.5 \%)$ included words from the immediately preceding sentence (see Section 6.5). On the other hand, without music, that rate was $41.3 \%$. This result shows that the rate of sentences including words from the immediately preceding sentence was higher with than without music. If the participant stuttered, we consider that the music distracted her attention from repeating her stereotypical utterances (see Section 6.5). The results indicate that MusiCuddle may give patients an opportunity to stop repeating utterances.

In the following example, a sentence changed into a completely different sentence when music was not presented (without music):

P: mazu imasendesu oyatsuja mazuyasumi (without music)

The following is an example in which a sentence included the word from the immediately preceding sentence when music was presented (with music):

P: mazugohandesu mazu... [ ] (Start music) (Stop music)

\section{Discussion}

The results of our case study suggest that the tunes presented by MusiCuddle may provide patients with an opportunity to stop repeating stereotypical utterances. The participant stuttered when each tune was presented. We believe that the music might shift the participant's interest to music from the repetition of stereotypical utterances.

In the case study, most of the first notes of the 50 presented tunes were within octave, D3 D4, and that of one tune was a much higher note, G\#4 (see Table 3). The F0 values derived from the participant's utterances were varied and wide-ranging. The pitch of the first note affects and changes the entire mood of the music (see Fig. 7). Tonality and pitch (high vs. low) are key determinants of musical mood. Therefore, it may be said that the MusiCuddle can play music to resonate with his/her mood by converting her utterance into pitch in accordance with the isoprinciple.

Moreover, the participant's repetitive stereotypical utterances were rhythmical, and most of her sentences fit into four-four time (see Fig. 9), like songs. Each tune presented by MusiCuddle began with a note on the F0 of the participant's utterances. However, each presented melody was different from the pattern of the participant's utterances.

On the other hand, in the preliminary experiment, when one of the authors repeated the participant's sentence using the same melody and rhythmic pattern, the participant kept repeating the same sentence, although she did pay attention to the author (see Section 6.3).

These results suggest that the sounds presented to patients should be neither identical to nor entirely different from their utterances. Patients might attend to the tunes according to their similarity in pitch and their attention may be deflected away from their repetitive stereotypical utterances if the melody is too strikingly different from their utterances.

In addition, in most music therapies for elderly people, therapists use reminiscence-focused music. In this examination, the participant sang part of a school song, "Yuki," twice. However, how the kinds of tunes presented influenced patient behavior could not be determined in the present experiment. These results suggest that music suitable for elderly patients with dementia is not limited to school songs when MusiCuddle is employed.

\section{Conclusion}

In this paper, we presented a music therapy system, "MusiCuddle," that semi-automatically plays a short musical phrase (tune) that overlaps with a patient's utterances and/or screaming. MusiCuddle extracts F0 values from patient's utterances and selects the tune; the first note is the same as the patient's F0. We performed a case study in which one of the authors used MusiCuddle to present tunes to a patient with dementia who repeated stereotypical utterances. When the tunes were presented, the patient tended to utter words from her preceding sentence at a higher rate than she did without music. This result suggested that the patient's attention might shift from her repetitive stereotypical utterances to the music. Furthermore, pitches extracted from the patient's utterances were varied and wide-ranging. The pitches of the first notes affect and change the entire mood of the music. This function of MusiCuddle may resonate with the patient's mood, in accordance with the iso-principle.

We performed a case study on only one participant. Although we simply say "dementia" when referring to deterioration of cognitive abilities, the individual expression and progression of symptoms differs from person to person. We have to grasp the patients' conditions and the advice of their primary doctors when we examine them. Thus, we will accumulate case studies of sev- 
eral participants in a through manner.

In the future, we will generate and compile more music data on the basis of the iso-principle and the findings of our research. Moreover, we will add the function that the system automatically transposes tunes into another key.

Acknowledgments This work was supported by Grants-inAid for Scientific Research (23-40168).

\section{References}

[1] Ministry of Health, Labour and Welfare: Degree of independent daily life in the elderly people with dementia (2012), available from 〈http://www.mhlw.go.jp/stf/houdou/2r9852000002iau1-att/ 2r9852000002iavi.pdf $\rangle$.

[2] Park, H. and Pringle Specht, J.K.: Effect of individualized music on agitation in individuals with dementia who live at home, $J$ Gerontol Nurs., Vol.35, No.8, pp.47-55 (2009).

[3] Svansdottir, H.B. and Snaedal, J.: Music therapy in moderate and severe dementia of Alzheimer's type: A case-control study, International Psychogeriatrics, Vol.18, pp.613-621 (2006).

[4] Nair, B.K., Heim, C., Krishnan, C., D'Este, C., Marley, J. and Attia, J.: The effect of Baroque music on behavioural disturbances in patients with dementia, Australasian Journal on Ageing, Vol.30, No.1, pp.11-15 (2011).

[5] Altshuler, I.M.: The past, present and future of musical therapy, Podolsky, E. (Ed.): Music therapy, pp.24-35, Philosophical Library (1954).

[6] Koen, B., Lloyd, J., Barz, G. and Smith, K.B.: The Oxford Handbook of Medical Ethnomusicology, Oxford University Press (2008).

[7] Michel, D.E. and Pinson, J.: Music Therapy In Principle And Practice, Charles C Thomas Publisher (2005).

[8] Grocke, D. and Wigram, T.: Receptive Methods in Music Therapy: Techniques and Clinical Applications for Music Therapy Clinicians, Educators and Students Jessica Kingsley Publishers (2007).

[9] Wigram, T.: Improvisation: Methods and Techniques for Music Therapy Clinicians, Educators and Students Jessica Kingsley Publishers (2004).

[10] de Klerk-Rubin, V.: Validation Techniques for Dementia Care: The Family Caregiver's Guide to Improving Communication, Health Professions Press, Baltimore (2007).

[11] Olsen, R.V., Hutchings, B.L. and Ehrenkrantz, E.: "Media Memory Lane" Interventions in an Alzheimer's Day Care Center, American J Alzheimer's Disease, Vol.15, No.3 (May/June 2000).

[12] Topo, P. et al.: Assessment of a music-based multimedia program for people with dementia, Dementia, Vol.3, No.3, pp.331-350 (2004).

[13] Kuwahara, N., Kuwabara, K., Abe, S., Yasuda, K. and Tetsutani, N.: Semantic Synchronization: Producing Effective Reminiscence Videos for Dementia Sufferers, 4th International Semantic Web Conference Poster E Demo Paper, PID-14 (2005).

[14] Alm, N. et al.: Living in the moment: An interactive entertainment system for people with dementia, Proc. CPAC2006, pp.30-36 (2006).

[15] Riley, P., Alm, N. and Newell, A.: An interactive tool to promote musical creativity in people with dementia, Computers in Human Behavior, Vol.25, pp.599-608 (2009).

[16] Imai, Y. and Hasegawa, K.: The Revised Hasegawa's Dementia Scale (HDS-R) - Evaluation of its Usefulness as a Screening Test for Dementia, Hong Kong J Psychiatr., Vol.4, No.2, pp.20-24 (1994).

[17] Itou, N. and Nishimoto, K.: A Voice-to-MIDI System for Singing Melodies with Lyrics, Proc. Int. Conf. ACE'07, pp.183-189, Salzburg (2007).

[18] Wakao, Y. and Okazaki, K.: A handbook of impromptu for music therapy, Ongaku-no-tomosya (1996). (in Japanese).

[19] Ashida, S.: The effect of reminiscence music therapy sessions on changes in depressive symptoms in elderly persons with dementia, Journal of Music Therapy, Vol.37, pp.170-182 (2000).

[20] Folstein, M.F., Folstein, S.E. and McHugh, P.R.: Mini-Mental State: A practical method for grading the cognitive state of patients for the clinician, Journal of Psychiatric Research, Vol.12, pp.189-198 (1975).

[21] Sherwood, S.J., Morris, J., Mor, V. and Gutkin, C.: Compendium of measures for describing and assessing long-term care populations, Hebrew Rehabilitation Center for the Aged, Boston, MA (1977).

[22] Shiba, R. and Nemoto, I.: The brain activities evoked by musical intervals in an octave, report of Research Center for Advanced Technologies, No.242, pp.19-22 (2006). (in Japanese).

[23] Smith, L.D. and Williams, R.N.: Children's artistic responses to musical intervals, American Journal of Psychology, Vol.112, pp.383-419 (1999).

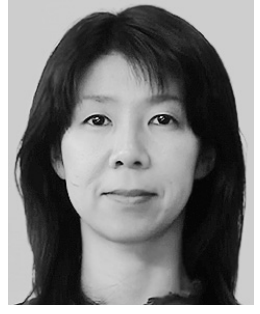

Chika Oshima was born in 1973. She is a postdoctoral researcher of the Japan Society for the Promotion of Science (RPD) since 2011. She received her B.M. degree from Musashino Academia Musicae in 1996. She received her M.S. and Ph.D. from Japan Advanced Institute of Science and Technology in 2001 and 2004, respectively and worked for Advanced Telecommunications Research Institute International (ATR) from 2004 to 2009 and for National Institute of Information and Communications Technology (NICT) from 2006 to 2010. She received Best Paper Award of ACM Multimedia 2004, IPSJ Yamashita SIG Research Award in 2006, Best Multi-disciplinary Paper Award of ICOST 2011, and Best Paper Award of IARIA in 2012. Her research interests include how to support communication between people mediated by computer and music.

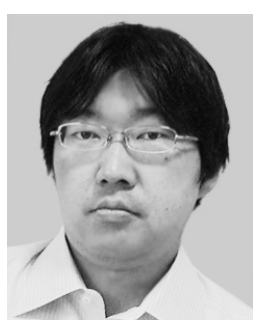

Naoki Itou was born in 1978. He received his M.E. degree from Kanagawa Institute of Technology in 2004. He completed the doctoral course of Japan Advanced Institute of Science and Technology and entered Intermedia Planning, inc. in 2011. He received his Ph.D. from JAIST in 2013. He received Best Multidisciplinary Paper Award of ICOST 2011, and Best Paper Award of IARIA in 2012. His research interests are musical signal processing and successful leading in project management. He is a member of IPSJ and IEICE.

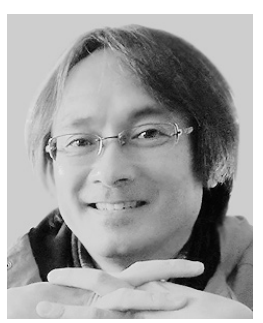

Kazushi Nishimoto is a professor and director of Research Center for Innovative Lifestyle Design at Japan Advanced Institute of Science and Technology since 1999. He received his B. Eng. and M. Eng. from Kyoto University, Japan, in 1985 and 1987, respectively, and received Dr. Eng. from Osaka University, Japan, in 1998. He worked for Matsushita Electric Industrial Co., Ltd. from 1987 till 1995, and ATR from 1992 till 2006. He was a researcher of Precursory Research for Embryonic Science and Technology of JST from 2000 till 2003, and a creator of Exploratory IT Human Resource Project of IPA, Japan, from 2006 till 2007. He received JSAI Anniversary Best Paper Award in 1999, IPSJ Sakai Memorial Award in 1999, Best Paper Award of ACM Multimedia 2004, Best Multi-disciplinary Paper Award of ICOST 2011, Best Paper Award of IARIA in 2012, and so on. His research interests include creativity mining, informal communication support, and human computer interaction. He is a member of IEEE computer society, ACM, JSAI and HIS. 


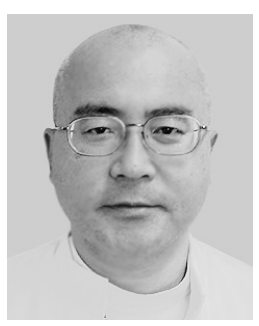

Kiyoshi Yasuda is a Speech/language therapist at the Rehabilitation department, Chiba Rosai Hospital since 1983. In 2003, he received his Ph.D. from Chiba University. In 2003 , he became a visiting researcher at Intelligent Robotics \& Communications Laboratories, ATR. From 2009, he is a project professor, Holistic Prosthetics Center, Kyoto Institute of Technology. He is a member of Japanese Society for Dementia Care, and Japanese society of Higher Brain Function Research. He received Best Multidisciplinary Paper Award of ICOST 2011 and Best Paper Award of IARIA in 2012. His major research themes include: development of Memory aids for people with memory-disabled and dementia, development of communication aid for people with aphasia, and comprehension of proper names and discourse in people with aphasic.

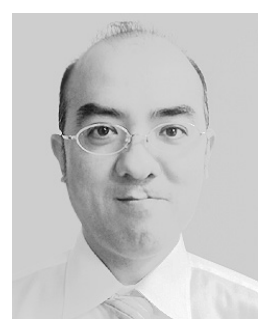

Naohito Hosoi was born in 1968. He is a designated psychiatrist and the director of Medical Center for Dementia at Sodegaura Satsukidai Hospital since 2006. He graduated in medicine from Toyama Medical and Pharmaceutical University and entered department of psychiatry at Chiba University in 1994. He has been engaged in Sodegaura Satsukidai Hospital since 1996. He has been in charge of the dementia treatment ward and the day-care facility for severe dementia since 2000. He is a member of Japanese Psychogeriatric Society and Japan Society for Dementia Research. $\mathrm{He}$ is a committee of Chiba-prefecture Council for the Promotion of measure against Dementia. He received Best Multidisciplinary Paper Award of ICOST 2011 and Best Paper Award of IARIA in 2012.

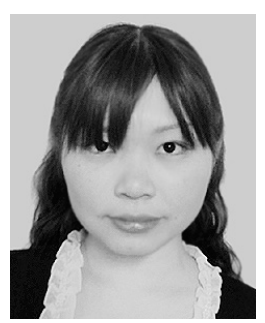

Hiromi Yamashita was born in 1987. She is a public health nurse. She received Masters of Nursing Science, Conferred from Saga University in 2013. She has been working in Chiba prefecture as a city employee (PHN) since 2013. She received Best Paper Award of IARIA in 2012.

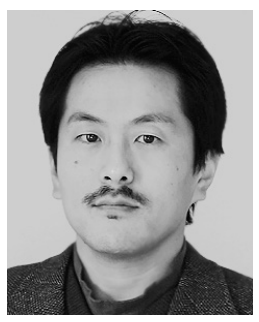

Koichi Nakayama was born in 1978. He received his $\mathrm{Ph} . \mathrm{D}$. in Informatics from Kyoto University in 2005. He is an associate professor at Graduate School of Science and Engineering, Saga University. His research interests include machine learning, optimization algorithm, complex systems and emergence system. He received Best Multi-disciplinary Paper Award of ICOST 2011 and Best Paper Award of IARIA in 2012.

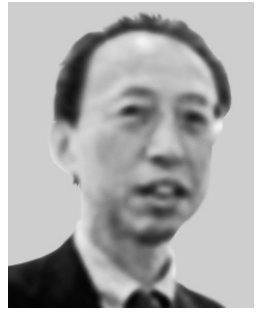

Etsuo Horikawa $\mathrm{He}$ is a professor of Cognitive Neuropsychology, Brain \& Mobility Laboratory, Graduate School of Medicine, Saga University. He earned his 1st Ph.D. degree on Information Biology and 2nd degree on Geriatric Medicine both from Tohoku University. He was a visiting scholar at Geriatric Medicine of University of Michigan. His research interests include detecting prodromal symptoms in early stage of Alzheimer's Disease, and Mild Cognitive Impairment, prevention of accidental falls in elderly people, fit to drive and cognitive rehabilitation. $\mathrm{He}$ is a member of the Gerontological Society of America, America Geriatric Society, International Neuropsychological Society and World Federation of Neurorehabilitation. He received Best Paper Award of IARIA in 2012. 